\title{
Mitochondrial Kv1.3: a New Target in Cancer Biology?
}

\author{
Vanessa Checchetto Elena Prosdocimi Luigi Leanza \\ Department of Biology, University of Padova, Padova, Italy
}

\section{Key Words}

Potassium channel $\bullet$ Mitochondria $\cdot$ Kv1.3 $\cdot$ Cancer $\cdot$ Signaling

\begin{abstract}
Kv1.3 is a voltage gated potassium channel located in the plasma membrane, as well as at intracellular levels, such as mitochondria (mitoKv1.3), nucleus and Golgi apparatus. The plasma membrane channel has been shown to be important for cell proliferation, while the mitochondrial counterpart has been related to modulation of cell death. Moreover, altered expression of Kv1.3 was observed in various tumors and Kv1.3 seems to be involved in development and progression of various cancerous forms. Recent experimental evidences have proved that pharmacological inhibition of the mitoKv1.3 succeeded in reducing up to $90 \%$ of tumor volume in vivo in orthotopic mouse model. Furthermore, mitoKv1.3 modulation could impact on cell proliferation as well as on regulation of intracellular signaling pathways. Indeed, the treatment with sub-lethal doses of mitoKv1.3 inhibitors can downregulate Wnt- $\beta$ catenin signaling by reducing mitochondrial ATP production and triggering ER-stress. In this review, we describe the role of the mitoKv1.3 in cell death, cancer and intracellular signaling. We will discuss how pharmacological modulation of mitochondrial potassium fluxes impact on mitochondrial membrane potential, reactive oxygen species production and ATP synthesis. All these changes in mitochondrial fitness are related to cell proliferation as well as to cell death and finally on cancer development and progression, so Kv1.3 (and mitoKv1.3) could be now considered a new oncological target.

\section{Introduction}

Mitochondria are intracellular organelles mainly involved in cellular respiration, but they can mediate also other processes, such as the regulation of differentiation, apoptosis, cell growth and cell cycle. The participation of mitochondria in these cellular processes seems to largely depend on ATP and ROS production, both generated during oxidative phosphorylation. Targeting mitochondrial metabolism by molecules able to specifically disrupt mitochondrial fitness and trigger cell death exclusively in cancer cells has become a promising strategy against cancer [1]. 


\section{Cellular Physiology Cell Physiol Biochem 2019;53(S1):52-62 \\ \begin{tabular}{ll|l} 
and Biochemistry & $\begin{array}{l}\text { DOl: 10.33594/000000195 } \\
\text { Published online: 20 December 2019 }\end{array}$ & $\begin{array}{l}\text { O 2019 The Author(s). Published by } \\
\text { Cell Physiol Biochem Press GmbH\&Co. KG }\end{array}$ \\
\cline { 2 - 3 } &
\end{tabular} \\ Chechetto et al.: MitoKv1.3 and Cancer}

Ion channels are ubiquitous pore-forming proteins, that control the passive diffusion of ions across cell membranes. Kv1.3 is a member of a large family of mammalian potassium $\left(\mathrm{K}^{+}\right)$channels, it is encoded by KCNA3, an intronless gene that is clustered together with KCNA2 and KCNA10 genes on chromosome 1 and its product is 575 amino acids long protein [2]. Kv1.3 (also known as MK3, HGK5, HLK3, PCN3, HPCN3 or HUKIII) is a voltagegated $\mathrm{K}^{+}$channel belonging to the Shaker related (Kv1, KCNA) 4x6 trans-membrane (TM) subfamily. 4x6TM structure refers to the common design of ion channels that contain four homologous repeat domains with six transmembrane $\alpha$-helices (S1-S6) in each domain. Furthermore, each domain contains a voltage-sensing element (S1-S4 helices) and a pore element (S5-S6 helices and the P-loop between S5 and S6). These functional parts of the protein respond to changes in membrane potential with a conformational change. Structural rearrangement of S4 domain are translated into a displacement of the S4-S5 linker that opens or closes the conduction pathway [3-5]. The NH2- and the COOH-terminal domains are both cytoplasmic and occur in channel clustering and in cell signaling via protein-protein intracellular interaction domains [6-13]. Kv1.3 currents have been originally recorded in the plasma membrane (PM) of human T-lymphocytes using patch-clamp technique [14]. The PM Kv1.3 channel displays an activation threshold between $50 \mathrm{mV}$ and $60 \mathrm{mV}$, a singlechannel conductance of $24 \mathrm{pS}$ that is activated by depolarization [15]. Kv1.3 channel is the main $\mathrm{K}^{+}$channel in T-lymphocytes, but today it is clear that it is expressed in many other tissues and cell types such as macrophages, microglia cells, natural killer cells, osteoclasts, platelets, central nervous system, olfactory bulb and testis [16-20]. Kv1.3, as well as other $\mathrm{Kv}$ channels, is characterized by a multiple subcellular localization: in PM, in inner mitochondrial membrane (IMM) (mitoKv1.3) [21, 22], in nuclear membrane [23] and in cisGolgi apparatus [24]. PM Kv1.3 is mainly involved in the tuning of cellular resting potential, in the regulation of proliferation and cell volume. The nuclear membrane Kv1.3 seems to contribute to regulate transcription and in the Golgi apparatus the role is not clear, while the contribution of mitoKv1.3 to cellular physiology is better studied. In the IMM, the roles played by mitoKv1.3 are multiple, ranging from regulation of mitochondrial membrane potential, of mitochrondrial volume, or of reactive oxygen species (ROS) production. Interestingly, a crucial role for this channel in apoptosis became evident, first in lymphocytes and later in other systems as well.

In this review we will focus on the role of mitoKv1.3 in modulating cell death by inducing changes in mitochondrial membrane potential, in ROS production and triggering of cell death in the context of cancer development and treatment.

\section{MitoKv1.3 features}

MitoKv1.3 is a $\mathrm{K}^{+}$ion channel through the IMM, which underlies the primary function of mitochondrial $\mathrm{K}^{+}$channels. It contributes to the regulation of the mitochondrial matrix volume, the respiratory rate, the mitochondrial membrane potential $(\Delta \Psi)$, the ROS synthesis and the redox signaling. In other words, it preserves mitochondrial metabolism and energy homeostasis. Biophysical and pharmacological analyses of channel activity and the generation of genetically modified cells have permitted to define the function of this protein. The IMM Kv1.3 localization has been demonstrated, initially, in T-lymphocytes [21], then also in macrophages $[19,25]$, in post-synaptic medial nucleus of the trapezoid body neurons [26], in hippocampal neurons [27] and in cancer cells lines PC-3 prostate tumor, MCF-7 breast adenocarcinoma, SAOS-2 osteosarcoma, in B16F10 melanoma cells and in primary B cells from Chronic Lymphocytic Leukemia (B-CLL) patients [15, 28, 29].

MitoKv1.3 is codified by the same gene as PM Kv1.3 and from a structural point of view, no differences have been indicated with respect to that of PM, however from a functional point of view the two channels appear to have very different behaviors. MitoKv1.3 is active at the extremely negative mitochondrial resting membrane potential $(-180,-200 \mathrm{mV})$ [21] and allows the influx of $\mathrm{K}^{+}$through the IMM, unlike PM Kv1.3 which opens in response to PM 
depolarization and mediates efflux of $\mathrm{K}^{+}$ions from the cytoplasm. Patch clamp experiments in gerbil hippocampal mitochondria demonstrated that mitoKv1.3 channel is voltage dependent in the $-50 /+50 \mathrm{mV}$ voltage range and shows a conductance of $109 \mathrm{pS}$ in $150 \mathrm{mM}$ $\mathrm{KCl}$ medium, sensitive to $10 \mathrm{nM}$ margatoxin (MgTx) [27]. MitoKv1.3 biophysical features have been investigated principally studying the pharmacological features of Kv1.3 channels using the known inhibitors. Kv1.3 blockers can be divided in two categories: I) PM non-permeable, such as TEA, 4-AP, benzamide, Margatoxin (MgTx), Charybdotoxin (ChTx) and Stichodactyla Toxin (ShK); II) PM-permeable, like psoralens and their derivatives (5-(4-phenylobutoxy) psoralen (Psora-4), 5-(4-phenoxybutoxy) psoralen (PAP-1) and its derivatives) and the rhinophenazine clofazimine (N,5-bis(4-chlorophenyl)-3-(1-methylethylimino)-5Hphenazine-2-amine), already used for autoimmune disorders [30, 31]. Only the second class are able to cross the PM and to block the mitoKv1.3 channels.

\section{MitoKv1.3, ROS production and apoptosis}

The reactive oxygen species (ROS) are produced mainly by mitochondria as a normal product of cellular respiration under normal physiological and pathological conditions and may have both beneficial and damaging role. Low levels of ROS production are needed to maintain physiological functions, like proliferation, host defense, signal transduction, and gene expression [32].

In healthy condition ROS concentration is low, while in cancer cells the ROS are increased because there is a reduced ability for ROS scavenging [33], and ROS species are necessary for induction of cancer proliferation, migration, and metastasis. As mentioned before, the inhibition of mitoKv1.3 channels triggers a transient hyperpolarization of the IMM which impacts on the redox state of the respiratory chain complexes, so rising single electron leakage at complexes I and III to molecular oxygen and increasing superoxide anion production. By oxidation of cysteine residues, ROS stimulates the mitochondrial permeability transition pore (PTP) that provokes the loss of the mitochondrial membrane potential and the mobilization and the release of cytochrome $\mathrm{c}$ from the mitochondrial cristae through the PTP and the outer mitochondrial membrane (OMM) voltage-dependent anion channels (VDAC), conclusively inducing the activation of the intrinsic apoptotic cascade [33-36]. The ROS production is then exponentially sustained also by the ROS induced-ROS release [37].

Apoptosis is a fundamental mechanism for normal cell development and maintenance of the cellular homeostasis. This pathway is controlled by proteins of the Bcl-2 family, which contains both pro-apoptotic and anti-apoptotic members and balances the decision between life and cell death [38]. Recent experimental evidences have highlighted how ion channels, particularly mitoKv1.3, play a crucial role in induction of programmed cell death. Mouse and human cells, genetically missing of channel Kv1.3 or transfected with siRNA that suppresses channel expression, are resistant to apoptosis induced by various stimuli, including overexpression of Bax [34]. Transfection of these cells with a specifically mitochondrial vector expressing Kv1.3 channel in this organelle restores the sensitivity of these cells to proapoptotic stimuli [34].

Patch clamp and co-immunoprecipitation experiments have confirmed the physical interaction between the channel and the pro-apoptotic Bcl-2 family member Bax after induction of programmed cell death. Incubation of isolated Kv1.3-positive mitochondria with Bax resulted in mitochondrial hyperpolarization compatible with inhibition of depolarizing influence of $\mathrm{K}^{+}$charges mediated by mitoKv1.3. A structural model foresees the insertion of the Bax monomer inside the OMM [39], where the alpha helices 5 and 6 protrude inside the mitochondrial inter membrane space (IMS), before the oligomerization and permeabilization of the membrane. The highly preserved amino acid lysine in position 128, present between these two helices of Bax, exposed in the mitochondrial IMS, interacts with the negative charges present in the vestibule of the channel pore. To support this evidence, it has been observed that in anti-apoptotic Bcl-2 family member proteins lysine 128 is replaced by a 


\section{Cellular Physiology Cell Physiol Biochem 2019;53(S1):52-62 \\ \begin{tabular}{ll|l} 
and Biochemistry & $\begin{array}{l}\text { DOI: 10.33594/000000195 } \\
\text { Published online:20 December } 2019\end{array}$ & $\begin{array}{l}\text { C } 2019 \text { The Author(s). Published by } \\
\text { Cell Physiol Biochem Press GmbH\&Co. KG }\end{array}$
\end{tabular} \\ Chechetto et al.: MitoKv1.3 and Cancer}

negatively charged amino acid. Furthermore, a mutation of lysine 128 in Bax does not allow contact between Bax and mitoKv1.3 and prevents the activation of apoptosis, transforming the pro-apoptotic protein Bax in an anti-apoptotic one. On the same line, the insertion of a lysine in position 158 in place of a glutamic acid in the anti-apoptotic protein Bcl-xL, turned this protein to pro-apoptotic $[36,40]$.

The use of membrane-permeant inhibitors of Kv1.3 channel can be used to mimic Bax inhibition and to induce the intrinsic apoptotic pathway in cancer cells. This mechanism has been demonstrated to be selective for malignant cells. This is due to a combination of elevated ROS production induced by mitoKv1.3 channel inhibition and the high basal ROS levels present in cancer cells. This increase leads the cells to bypass the ROS critical threshold to trigger cell death. These events do not occur in case of normal cells since a lower amount of ROS are produced, and even if mitoKv1.3 inhibition happens the increase of ROS production is not sufficient to reach the critical threshold and induce cell death. It is importantly to note that if mitoKv1.3 is missing, even the induction of a mild oxidative stress does not sensitize cells to apoptosis, demonstrating a synergistic role of both mitoKv1.3 expression and cellular redox state in this pathway. Therefore, normal cells do not undergo apoptosis upon application of the inhibitors [29, 41].

\section{MitoKv1.3 and cancer}

In general, Kv1.3 is involved in several pathological manifestations, such as the onset of autoimmune diseases, chronic diseases and in the development and progression of various cancerous forms [42]: melanoma [43], breast adenocarcinoma [44], pancreatic adenocarcinoma (PDAC) [45], glioblastoma [46] and Chronic Lymphocytic Leukemia (B-CLL) [47]. In some cancerous forms there is an altered expression of specific ion channels, evidence that tumours constitute a form of channelopathy [48]. However, the altered expression of Kv1.3, observed in various tumours, does not present a unique and isolable pattern: sometimes there is an overexpression, as for example in breast adenocarcinoma [44], while, on the contrary, it is expressed in a very low amount on other occasions, as in the case of prostate cancer [49].

Kv1.3 expression can also change during cancer progression; considering the above examples, i.e. the case of breast adenocarcinoma, it has been observed (through the comparison of the expression levels of Kv1.3 mRNA of different stages compared to normal tissues) that there is an overexpression in the first stage of this breast cancer. Conversely, at the level of the second and third stage, the level of mRNA is not very different from the physiological levels, however, going to rise dramatically during the last stage of the tumour and in the metastatic phase [23].

Despite the evidence gathered, there is no reliable model yet that explains the correlation between the expression of the Kv1.3 channel and these tumours: in various types of cancer, such as B-CLL, it is possible to observe the up-regulation of the channel, which seems to be caused by the haploinsufficiency of a gene regulating the channel, the KCNRG [29]. Similarly, inhibition of B-RAF kinase by a pro-apoptotic and anti-proliferative agent (PLX-4720) has been shown to reduce channel expression in B-CLL but not in normal B cells [47]. This evidence suggests that B-RAF-dependent activity may be related to channel overexpression in this type of cancer cell: similarly channel reduction in some types of cancer, such as pancreatic adenocarcinoma [44] and colon cancer [50], seems to be related to an altered methylation of the channel promoter gene, which is particularly pronounced in metastatic cells.

According to differential expression of the channel during cancer development, its pharmacological targeting was studied in the latest years. Indeed, recent studies have highlighted the particular importance of mitoKv1.3 as a new specific oncological target [51]: indeed, as stated above there are some membrane permeant inhibitors of the channel Kv1.3, such as Psora-4, PAP-1 and clofazimine, capable to induce apoptosis only in Kv1.3 


\section{Cellular Physiology Cell Physiol Biochem 2019;53(S1):52-62 \\ \begin{tabular}{ll|l} 
and Biochemistry & $\begin{array}{l}\text { DOI: 10.33594/000000195 } \\
\text { Published online: } 20 \text { December } 2019\end{array}$ & $\begin{array}{l}\text { C } 2019 \text { The Author(s). Published by } \\
\text { Cell Physiol Biochem Press GmbH\&Co. KG }\end{array}$
\end{tabular}

expressing cancer cells, sparing healthy one not expressing the channel [29]. The activation of the intrinsic pathway of cell death, due to the inhibition of mitoKv13 in these cells, is characterized by increased ROS production, depolarization of the internal mitochondrial membrane, release of cytochrome c, cleavage of polyADP ribose-polymerase (PARP), followed by activation of caspases 9 and 3 (Fig. 1) [28, 29].

Lowering channel expression with siRNA demonstrated that membrane permeant inhibitors were ineffective in inducing programmed cell death [28]. It is therefore clear that the expression of mitoKv1.3 is necessary to produce all those mitochondrial modifications that end with apoptosis. On the contrary, Kv1.3 inhibitors that do not permeate the plasma membrane and therefore target only PM Kv1.3, e.g. the toxins MgTx, ShK and ChTx, do not induce the same apoptotic events [28]. It is important to note that programmed cell death is also induced in cancer cells expressing Kv1.3 without the pro-apoptotic proteins Bax and Bak, as demonstrated in the case of Jurkat leukemic cells and murine embryonic fibroblasts (MEF), or overexpressing the anti-apoptotic protein Bcl-2 or a mutated form of p53, as observed in B-CLL cells $[28,29]$.

Kv1.3 membrane permeant inhibitors have also been shown to be effective in vivo, in orthotopic mouse models. The treatment with clofazimine was able to reduce the size of melanoma and PDAC by $90 \%$ and $50 \%$ respectively $[28,45]$, showing no other alterations in the other healthy organs of the mice.

Recently, new mitochondria-targeted PAP-1 derivatives, namely PAPTP, PCARBTP and PCTP, have been synthesized by the addition of a positively charged lipophilic group (TPP ${ }^{+}$ triphenylphoshonium), attached to the psoralene chain of PAP-1 [52,53]. These new positively charged compounds accumulate more easily and very fast within the mitochondria, which display a negative charge at the level of the IMM. The mitochondriotropic characteristics of these compounds target them specifically to the mitoKv1.3. PAPTP, PCARBTP and PCTP are able to activate the mitochondrial dependent programmed cell death specifically only in cancer cells expressing mitoKv1.3, sparing healthy cells. The molecular pathway recapitulates all the mitochondrial features already demonstrated for membrane permeant Kv1.3 inhibitors: I) initial hyperpolarization of the IMM caused by the block of the $\mathrm{K}^{+}$fluxes; II) chemical reduction (not direct inhibition) of the respiratory chain complexes which alter mitochondrial respiration and ATP production; III) production of mitochondrial ROS; IV) activation of the PTP that lead to IMM depolarization; V) release of cytochrome $c$ and induction of intrinsic apoptosis [53]. Importantly, these results have been obtained by experiments in vitro, involving different cancer cell lines, ex-vivo, studying the effects of these new synthetized derivatives on B-CLL from patients and healthy subjects, and in vivo, using orthotopic mouse melanoma and PDAC models. In the latter studies, PAPTP and PCARBTP were able to reduce tumor volume by $90 \%$ and $60 \%$, respectively for melanoma and PDAC, 


\section{Cellular Physiology Cell Physiol Biochem 2019;53(S1):52-62 \\ \begin{tabular}{ll|l} 
and Biochemistry & $\begin{array}{l}\text { DOI: 10.33594/000000195 } \\
\text { Published online: } 20 \text { December } 2019\end{array}$ & $\begin{array}{l}\text { C } 2019 \text { The Author(s). Published by } \\
\text { Cell Physiol Biochem Press GmbH\&Co. KG }\end{array}$
\end{tabular} \\ Chechetto et al.: MitoKv1.3 and Cancer}

without affecting healthy organs, immune system or cardiac function [53]. It is very important to note that also in this case apoptosis is not only due to over-expression of the channel in the mitochondrial membrane. The efficacy of these new compounds in combination with the addition of antioxidants is drastically reduced, further demonstrating that apart Kv1.3 expression also the production of ROS is a fundamental step of the pro-apoptotic process induced by these drugs. The release of ROS induces the activation of the hypoxia inducible factor (HIF1 $\alpha$ ) in the cytoplasm, which promotes tumour development and metastasis [33]. As stated above, the selective death of cancer cells is therefore due to the combination of two synergistic factors [53]: the selectivity of compounds to the mitoKv1.3 added to a basal level of ROS present in the mitochondria of cancer cells. Healthy cells are therefore spared and therefore do not experience programmed cell death [54].

\section{MitoKv1.3 in proliferation and cell signaling}

The cell cycle is constituted by four phases: G1 (gap 1), the S (synthesis of DNA), the G2 (gap 2) and the $\mathrm{M}$ (mitosis). The progression among the different phases is finely regulated by the presence of different check points represented by the cyclic activation or inhibition of cyclin-dependent kinases (CDKs) [55]. $\mathrm{PM} \mathrm{K}^{+}$channels play an important role during cell proliferation, since they are involved in the regulation of plasma membrane potential and cell volume. Indeed, a high $\mathrm{K}^{+}$permeability hyperpolarizes plasma membrane and is necessary for the $\mathrm{G} 1$ to $\mathrm{S}$ transition, while a reduced $\mathrm{K}^{+}$permeability induces membrane depolarization that is needed to proceed from the M to the G1 phase [55]. Among the $\mathrm{K}^{+}$channels, Kv1.3 was one of the first that have been demonstrated to be involved in controlling proliferation of different cell types, e.g. microglia, oligodendrocytes and macrophages $[55,56]$. Furthermore, Kv1.3 has been shown to be upregulated during G1 phase and its inhibition generally induces a block in proliferation $[57,58]$. Not only fluctuation in $\mathrm{K}^{+}$fluxes are important to regulate proliferation but also other modification, such as conformational changes or protein-protein interactions. According to some proposed models, the activation of Kv1.3 together with the $\mathrm{K}_{\mathrm{Ca}} 3.1\left(\mathrm{Ca}^{2+}\right.$-dependent $\mathrm{K}^{+}$channel activated by $\mathrm{Ca}^{2+}$-calmodulin) can hyperpolarize the plasma membrane, so reducing the $\mathrm{Ca}^{2+}$ driving force through the plasma membrane induced by the plasma membrane depolarization. This modulation of the plasma membrane potential is able to modulate $\mathrm{Ca}^{2+}$ influx inside the cells and in turn to regulate the $\mathrm{Ca}^{2+}$ dependent cellular processes, such as T-cell activation and proliferation [59]. Another model suggests that Kv1.3 undergoes conformational changes on its voltage domain following membrane depolarization and this variation is sufficient to induce a pro-proliferative activity of the channel [57]. Indeed, a Kv1.3 pore-less mutant favors proliferation independently on its voltage properties [60]. Moreover, Kv1.3 acts as a voltage sensor that is activated by depolarization, causing a modification of its C-terminal domain and exposing site for tyrosine and serine phosphorylation by members of the MEK-ERK signaling pathways [10, 49]. In this contest, we have demonstrated that B-RAF a member of the RAS-RAF-MEK-ERK signal transduction or mitogen-activated protein kinase (MAPK) pathway, involved in tuning cell proliferation, differentiation and survival in response to extracellular signals, mediates the up-regulation of Kv1.3 in B-CLL cells [47].

Recently, we have demonstrated that even mitoKv1.3 can modulate cell proliferation (Fig. 1). Indeed, PAPTP and PCARBTP at nanomolar concentration are able to enhance cell proliferation by increasing the percentage of PDAC cell lines in S phase, in contrast to the block of the cell cycle induced by membrane impermeant toxins, which act exclusively on PM Kv1.3 [61]. The molecular mechanism involves a mild ROS production known to promote cell proliferation [62-64] and it is completely reverted by the pre-treatment with antioxidants or ROS scavengers [61].

Finally, we recently demonstrated that pharmacological modulation of mitoKv1.3 by sub-lethal doses of PAPTP and PCARBTP is able to reduce the canonical Wnt- $\beta$ catenin signaling pathway [65]. Wnt signaling is an important pathway that can be involved in 


\section{Cellular Physiology Cell Physiol Biochem 2019;53(S1):52-62 \\ \begin{tabular}{ll|l} 
and Biochemistry & $\begin{array}{l}\text { DOl: 10.33594/000000195 } \\
\text { Published online: 20 December 2019 }\end{array}$ & $\begin{array}{l}\text { O 2019 The Author(s). Published by } \\
\text { Cell Physiol Biochem Press GmbH\&Co. KG }\end{array}$ \\
\cline { 2 - 3 } &
\end{tabular} \\ Chechetto et al.: MitoKv1.3 and Cancer}

cell proliferation and differentiation. When Wnt signaling is in the "off" state, $\beta$-catenin is constitutively phosphorylated by the so called "destruction complex" and sent to proteasomal degradation. Conversely, when this pathway is activated by the binding of a Wnt ligands on extracellular receptors, the destruction complex is disassembled and the unphosphorylated $\beta$-catenin is accumulated in the cytosol and it translocates in the nucleus where can activate the transcription of Wnt-related genes [66]. Aberrant activation of Wnt/ $\beta$-catenin signaling promotes the development of several cancers [67], by actively participating in conferring unlimited proliferative potential to these pathologic cells and leading to a worsened outcome in patients where this pathway is dysregulated [68]. Wnt signaling has also been shown to be involved in the Epithelial to Mesenchymal Transition (EMT) that drives cells towards differentiation into migratory mesenchymal and invasive cells, able to form metastasis. Thus, molecules/processes involved in this signaling pathway are considered attractive targets for the design of new chemotherapeutic agents, and some inhibitors recently entered clinical testing $[69,70]$. On this issue, we have recently demonstrated that Wnt signaling can be specifically downregulated in cancer cells by tuning mitochondrial function and ATP production. Our results demonstrated that variation of mitochondrial fitness by several drugs (among the others also sub-lethal doses PAPTP and PCARBTP) or by genetic alteration ultimately impacts on mitochondrial ATP synthesis [65]. This mitochondrial ATP depletion in the MAMs impacts on the sarco/endoplasmic reticulum $\mathrm{Ca}^{2+}$-ATPase (SERCA) activity, reducing $\mathrm{Ca}^{2+}$ uptake into the ER and in turn inducing ER stress, observed by an increased phosphorylation of the eukaryotic initiation factor 2 (EIF $2 \alpha$ ) and by the up-regulation of the activating transcription factor 4 (ATF4). This new paradigm has been also demonstrated in vitro in colorectal cancer cells. Finally, CHOP (CCAAT/enhancer-binding protein homologous protein)/GADD153 is able to modulate T cell factor/lymphoid enhancer factor (TCF/LEF) transcriptional activity so to decrease canonical Wnt pathway. This was the first evidence that the modulation of mitochondrial fitness (even by inhibiting a mitochondrial $\mathrm{K}^{+}$channel; Fig. 1) could modulate the activation of an intracellular signaling pathway.

\section{Conclusion}

In the latest years, ion channels are arising as new oncological targets. Several increasing evidences are describing the role of these proteins in cancer development as well as on its progression from primary tumor to metastasis. In this field, a growing role is on charge of the voltage-gated $\mathrm{K}^{+}$channel Kv1.3. Several observations have linked this channel and its activity to several pathological pathways. This channel has an altered expression in malignant cells than in healthy ones, its modulation could impact to some intracellular signaling pathways, such as the RAS or the Wnt signaling ones. Furthermore, these signaling pathways can in turn regulate Kv1.3 (and possibly mitoKv1.3) expression and function. Importantly, pharmacological targeting of Kv1.3 is well known and can be targeted by different drugs able to selectively inhibit the PM Kv1.3 or the intracellular one up to the mitoKv1.3, as observed by the newly designed mitochondria-targeted compounds. If we consider that this channel is not only involved in cancer development but also in other diseases, it is clear that future research on Kv1.3 will improve our knowledge of ion channels role in pathological cellular transformation. 


\section{Cellular Physiology Cell Physiol Biochem 2019;53(S1):52-62 \begin{tabular}{ll|l|l|}
\hline DOl: 10.33594/000000195 & () 2019 The Author(s). Published by \\
\hline
\end{tabular} and Biochemistry Published online: 20 December 2019 Cell Physiol Biochem Press GmbH\&Co. KG \\ Chechetto et al.: MitoKv1.3 and Cancer}

\section{Acknowledgements}

The authors thanks Prof. Ildikò Szabò for useful discussions and scientific support. The authors are grateful to Italian Association for Cancer Research (AIRC), to the EMBO, and to the Department of Biology of the University of Padova. The authors disclosed receipt of the following financial support for the research, authorship, and/or publication of this article: The research leading to these results has received funding from Italian Association for Cancer Research (AIRC) under MFAG 2019 - ID. 23271 project - P.I. Leanza Luigi; L.L. is the recipient of a young researcher grant (no. GRIC12NN5G) and a PRID 2017 (no. BIRD162511) from the University of Padova and is grateful to EMBO for a short-term fellowship (ASTF 233-2014). Author Contributions: L.L., V.C. and E.P. wrote the manuscript. The authors have no ethical conflicts to disclose.

\section{Disclosure Statement}

The authors have no conflicts of interest to declare.

\section{References}

1 Fulda S, Galluzzi L, Kroemer G: Targeting mitochondria for cancer therapy. Nat Rev Drug Discov 2010;9:447-464.

2 Folander K, Douglass J, Swanson R: Confirmation of the assignment of the gene encoding Kv1.3, a voltage-gated potassium channel (KCNA3) to the proximal short arm of human chromosome 1. Genomics 1994;23:295-296.

3 Binggeli R, Weinstein RC: Membrane potentials and sodium channels: hypotheses for growth regulation and cancer formation based on changes in sodium channels and gap junctions. J Theor Biol 1986;123:377401.

4 Jiang Y, Lee A, Chen J, Ruta V, Cadene M, Chait BT, MacKinnon R: X-ray structure of a voltage-dependent K+ channel. Nature 2003;423:33-41.

5 Pathak M, Kurtz L, Tombola F, Isacoff E: The cooperative voltage sensor motion that gates a potassium channel. J Gen Physiol 2005;125:57-69.

6 Strang C, Cushman SJ, DeRubeis D, Peterson D, Pfaffinger PJ: A central role for the T1 domain in voltagegated potassium channel formation and function. J Biol Chem 2001;276:28493-28502.

7 Pongs 0, Schwarz JR: Ancillary Subunits Associated With Voltage-Dependent K+ Channels. Physiol Rev 2010;90:755-796.

8 Marks DR, Fadool DA: Post-synaptic density perturbs insulin-induced Kv1.3 channel modulation via a clustering mechanism involving the SH3 domain. J Neurochem 2007;103:1608-1627.

9 Martinez-Marmol R, Perez-Verdaguer M, Roig SR, Vallejo-Gracia A, Gotsi P, Serrano-Albarras A, Bahamonde MI, Ferrer-Montiel A, Fernandez-Ballester G, Comes N, Felipe A: A non-canonical di-acidic signal at the C-terminus of Kv1.3 determines anterograde trafficking and surface expression. J Cell Sci 2013;126:56815691.

10 Jimenez-Perez L, Cidad P, Alvarez-Miguel I, Santos-Hipolito A, Torres-Merino R, Alonso E, de la Fuente MA, Lopez-Lopez JR, Perez-Garcia MT: Molecular Determinants of Kv1.3 Potassium Channels-induced Proliferation. J Biol Chem 2016;291:3569-3580.

11 Hajdu P, Martin GV, Chimote AA, Szilagyi O, Takimoto K, Conforti L: The C-terminus SH3-binding domain of Kv1.3 is required for the actin-mediated immobilization of the channel via cortactin. Mol Biol Cell 2015;26:1640-1651.

12 Pérez-Verdaguer M, Capera J, Martínez-Mármol R, Camps M, Comes N, Tamkun MM, Felipe A: Caveolin interaction governs Kv1.3 lipid raft targeting. Sci Rep 2016;6:22453.

13 Spear JM, Koborssy DA, Schwartz AB, Johnson AJ, Audhya A, Fadool DA, Stagg SM: Kv1.3 contains an alternative C-terminal ER exit motif and is recruited into COPII vesicles by Sec24a. BMC Biochem 2015;16:16. 


\section{Cellular Physiology Cell Physiol Biochem 2019;53(S1):52-62

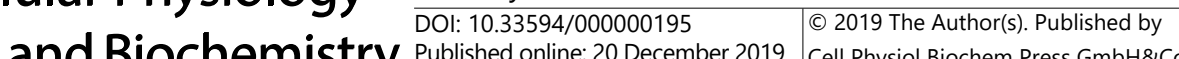 and Biochemistry Published online: 20 December 2019 Cell Physiol Biochem Press GmbH\&Co. KG \\ Chechetto et al.: MitoKv1.3 and Cancer}

14 Matteson DR, Deutsch C: K channels in T lymphocytes: a patch clamp study using monoclonal antibody adhesion. Nature 1984;307:468-471.

15 Szabo I, Zoratti M: Mitochondrial channels: ion fluxes and more. Physiol Rev 2014;94:519-608.

16 Kotecha SA, Schlichter LC: A Kv1.5 to Kv1.3 switch in endogenous hippocampal microglia and a role in proliferation. J Neurosci 1999;19:10680-10693.

17 Cayabyab FS, Khanna R, Jones OT, Schlichter LC: Suppression of the rat microglia Kv1.3 current by srcfamily tyrosine kinases and oxygen/glucose deprivation. Eur J Neurosci 2000;12:1949-1960.

18 Peng Y, Lu K, Li Z, Zhao Y, Wang Y, Hu B, Xu P, Shi X, Zhou B, Pennington M, Chandy KG, Tang Y: Blockade of Kv1.3 channels ameliorates radiation-induced brain injury. Neuro Oncol 2014;16:528-539.

19 Vicente R, Escalada A, Villalonga N, Texido L, Roura-Ferrer M, Martin-Satue M, Lopez-Iglesias C, Soler C, Solsona C, Tamkun MM, Felipe A: Association of Kv1.5 and Kv1.3 contributes to the major voltagedependent $\mathrm{K}+$ channel in macrophages. J Biol Chem 2006;281:37675-37685.

20 Comes N, Bielanska J, Vallejo-Gracia A, Serrano-Albarras A, Marruecos L, Gomez D, Soler C, Condom E, Ramon YCS, Hernandez-Losa J, Ferreres JC, Felipe A: The voltage-dependent K(+) channels Kv1.3 and Kv1.5 in human cancer. Front Physiol 2013;4:283.

21 Szabo I, Bock J, Jekle A, Soddemann M, Adams C, Lang F, Zoratti M, Gulbins E: A novel potassium channel in lymphocyte mitochondria. J Biol Chem 2005;280:12790-12798.

22 Zoratti M, De Marchi U, Gulbins E, Szabo I: Novel channels of the inner mitochondrial membrane. Biochim Biophys Acta 2009;1787:351-363.

23 Jang SH, Kang KS, Ryu PD, Lee SY: Kv1.3 voltage-gated K(+) channel subunit as a potential diagnostic marker and therapeutic target for breast cancer. BMB Rep 2009;42:535-539.

24 Zhu J, Yan J, Thornhill WB: The Kv1.3 potassium channel is localized to the cis-Golgi and Kv1.6 is localized to the endoplasmic reticulum in rat astrocytes. FEBS J 2014;281:3433-3445.

25 Leanza L, Zoratti M, Gulbins E, Szabò I: Induction of apoptosis in macrophages via Kv1.3 and Kv1.5 potassium channels. Curr Med Chem 2012;19:5394-5404.

26 Gazula VR, Strumbos JG, Mei X, Chen H, Rahner C, Kaczmarek LK: Localization of Kv1.3 channels in presynaptic terminals of brainstem auditory neurons. J Comp Neurol 2010;518:3205-3220.

27 Bednarczyk P, Kowalczyk JE, Beresewicz M, Dołowy K, Szewczyk A, Zabłocka B: Identification of a voltage-gated potassium channel in gerbil hippocampal mitochondria. Biochem Biophys Res Commun 2010;397:614-620.

28 Leanza L, Henry B, Sassi N, Zoratti M, Chandy KG, Gulbins E, Szabò I: Inhibitors of mitochondrial Kv1.3 channels induce Bax/Bak-independent death of cancer cells. EMBO Mol Med 2012;4:577-593.

29 Leanza L, Trentin L, Becker KA, Frezzato F, Zoratti M, Semenzato G, Gulbins E, Szabo I: Clofazimine, Psora-4 and PAP-1, inhibitors of the potassium channel Kv1.3, as a new and selective therapeutic strategy in chronic lymphocytic leukemia. Leukemia 2013;27:1782-1785.

30 Prosdocimi E, Checchetto V, Leanza L: Targeting the Mitochondrial Potassium Channel Kv1.3 to Kill Cancer Cells: Drugs, Strategies, and New Perspectives. SLAS Discov 2019;24:882-892.

31 Ren YR, Pan F, Parvez S, Fleig A, Chong CR, Xu J, Dang Y, Zhang J, Jiang H, Penner R, Liu JO: Clofazimine inhibits human Kv1.3 potassium channel by perturbing calcium oscillation in T lymphocytes. PLoS One 2008;3:e4009.

32 Dröge W: Free radicals in the physiological control of cell function. Physiol Rev 2002;82:47-95.

33 Sabharwal SS, Schumacker PT: Mitochondrial ROS in cancer: initiators, amplifiers or an Achilles' heel? Nat Rev Cancer 2014;14:709-721.

34 Szabo I, Bock J, Grassme H, Soddemann M, Wilker B, Lang F, Zoratti M, Gulbins E: Mitochondrial potassium channel Kv1.3 mediates Bax-induced apoptosis in lymphocytes. Proceedings of the National Academy of Sciences 2008;105:14861-14866.

35 Bachmann M, Costa R, Peruzzo R, Prosdocimi E, Checchetto V, Leanza L: Targeting Mitochondrial Ion Channels to Fight Cancer. Int J Mol Sci 2018;19:pii:E2060.

36 Gulbins E, Sassi N, Grassmè H, Zoratti M, Szabò I: Role of Kv1.3 mitochondrial potassium channel in apoptotic signalling in lymphocytes. Biochim Biophys Acta 2010;1797:1251-1259.

37 Zorov DB, Juhaszova M, Sollott SJ: Mitochondrial reactive oxygen species (ROS) and ROS-induced ROS release. Physiol Rev 2014;94:909-950.

38 Singh R, Letai A, Sarosiek K: Regulation of apoptosis in health and disease: the balancing act of BCL-2 family proteins. Nat Rev Mol Cell Biol 2019;20:175-193. 


\section{Cellular Physiology Cell Physiol Biochem 2019;53(S1):52-62 \begin{tabular}{ll|l|l|l}
\hline DOI: 10.33594/000000195 & (C)19 The Author(s). Published by
\end{tabular} and Biochemistry Published online: 20 December 2019 Cell Physiol Biochem Press GmbH\&Co. KG \\ Chechetto et al.: MitoKv1.3 and Cancer}

39 Annis MG, Soucie EL, Dlugosz PJ, Cruz-Aguado JA, Penn LZ, Leber B, Andrews DW: Bax forms multispanning monomers that oligomerize to permeabilize membranes during apoptosis. EMBO J 2005;24:2096-2103.

40 Szabò I, Soddemann M, Leanza L, Zoratti M, Gulbins E: Single-point mutations of a lysine residue change function of Bax and Bcl-xL expressed in Bax- and Bak-less mouse embryonic fibroblasts: novel insights into the molecular mechanisms of Bax-induced apoptosis. Cell Death Differ 2011;18:427-438.

41 Teisseyre A, Gąsiorowska J, Michalak K: Voltage-Gated Potassium Channels Kv1.3--Potentially New Molecular Target in Cancer Diagnostics and Therapy. Adv Clin Exp Med 2015;24:517-524.

42 Serrano-Albarrás A, Estadella I, Cirera-Rocosa S, Navarro-Pérez M, Felipe A: Kv1.3: a multifunctional channel with many pathological implications. Expert Opin Ther Targets 2018;22:101-105.

43 Artym VV, Petty HR: Molecular proximity of Kv1.3 voltage-gated potassium channels and beta(1)-integrins on the plasma membrane of melanoma cells: effects of cell adherence and channel blockers. J Gen Physiol 2002;120:29-37.

44 Brevet M, Haren N, Sevestre H, Merviel P, Ouadid-Ahidouch H: DNA methylation of K(v)1.3 potassium channel gene promoter is associated with poorly differentiated breast adenocarcinoma. Cell Physiol Biochem 2009;24:25-32.

45 Zaccagnino A, Managò A, Leanza L, Gontarewitz A, Linder B, Azzolini M, Biasutto L, Zoratti M, Peruzzo R, Legler K, Trauzold A, Kalthoff H, Szabo I: Tumor-reducing effect of the clinically used drug clofazimine in a SCID mouse model of pancreatic ductal adenocarcinoma. Oncotarget 2017;8:38276-38293.

46 Venturini E, Leanza L, Azzolini M, Kadow S, Mattarei A, Weller M, Tabatabai G, Edwards MJ, Zoratti M, Paradisi C, Szabò I, Gulbins E, Becker KA: Targeting the Potassium Channel Kv1.3 Kills Glioblastoma Cells. Neurosignals 2017;25:26-38.

47 Szabo I, Trentin L, Trimarco V, Semenzato G, Leanza L: Biophysical characterization and expression analysis of Kv1.3 potassium channel in primary human leukemic B cells. Cell Physiol Biochem 2015;37:965-978.

48 Litan A, Langhans SA: Cancer as a channelopathy: ion channels and pumps in tumor development and progression. Front Cell Neurosci 2015;9:86.

49 Teisseyre A, Palko-Labuz A, Sroda-Pomianek K, Michalak K: Voltage-Gated Potassium Channel Kv1.3 as a Target in Therapy of Cancer. Front Oncol 2019;9:933.

50 He T, Wang C, Zhang M, Zhang X, Zheng S, Linghu E, Guo M: Epigenetic regulation of voltage-gated potassium ion channel molecule Kv1.3 in mechanisms of colorectal cancer. Discov Med 2017;23:155-162.

51 Leanza L, Zoratti M, Gulbins E, Szabo I: Mitochondrial ion channels as oncological targets. Oncogene 2014;33:5569-5581.

52 Mattarei A, Romio M, Manago A, Zoratti M, Paradisi C, Szabo I, Leanza L, Biasutto L: Novel MitochondriaTargeted Furocoumarin Derivatives as Possible Anti-Cancer Agents. Front Oncol 2018;8:122.

53 Leanza L, Romio M, Becker KA, Azzolini M, Trentin L, Managò A, Venturini E, Zaccagnino A, Mattarei A, Carraretto L, Urbani A, Kadow S, Biasutto L, Martini V, Severin F, Peruzzo R, Trimarco V, Egberts JH, Hauser C, Visentin A, et al.: Direct Pharmacological Targeting of a Mitochondrial Ion Channel Selectively Kills Tumor Cells In vivo. Cancer Cell 2017;31:516-531.e510.

54 Trachootham D, Alexandre J, Huang P: Targeting cancer cells by ROS-mediated mechanisms: a radical therapeutic approach? Nat Rev Drug Discov 2009;8:579-591.

55 Urrego D, Tomczak AP, Zahed F, Stühmer W, Pardo LA: Potassium channels in cell cycle and cell proliferation. Phil Trans R Soc B 2014;369:20130094.

56 DeCoursey TE, Chandy KG, Gupta S, Cahalan MD: Voltage-gated K+ channels in human T lymphocytes: a role in mitogenesis? Nature 1984;307:465-468.

57 Serrano-Novillo C, Capera J, Colomer-Molera M, Condom E, Ferreres JC, Felipe A: Implication of VoltageGated Potassium Channels in Neoplastic Cell Proliferation. Cancers (Basel) 2019;11:pii:E287.

58 Amigorena S, Choquet D, Teillaud JL, Korn H, Fridman WH: Ion channel blockers inhibit B cell activation at a precise stage of the G1 phase of the cell cycle. Possible involvement of K+ channels. J Immunol 1990;144:2038-2045.

59 Cahalan MD, Chandy KG: The functional network of ion channels in T lymphocytes. Immunol Rev 2009;231:59-87.

60 Cidad P, Jiménez-Pérez L, García-Arribas D, Miguel-Velado E, Tajada S, Ruiz-McDavitt C, López-López JR, Pérez-García MT: Kv1.3 channels can modulate cell proliferation during phenotypic switch by an ion-flux independent mechanism. Arterioscler Thromb Vasc Biol 2012;32:1299-1307. 


\section{Cellular Physiology Cell Physiol Biochem 2019;53(S1):52-62

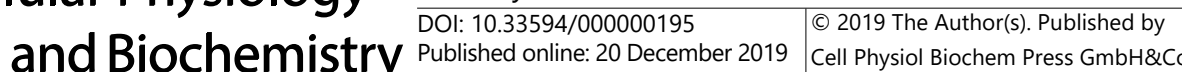 \\ Chechetto et al.: MitoKv1.3 and Cancer}

61 Peruzzo R, Mattarei A, Romio M, Paradisi C, Zoratti M, Szabò I, Leanza L: Regulation of Proliferation by a Mitochondrial Potassium Channel in Pancreatic Ductal Adenocarcinoma Cells. Front Oncol 2017;7:239.

62 Diebold L, Chandel NS: Mitochondrial ROS regulation of proliferating cells. Free Radic Biol Med 2016;100:86-93.

63 Ralph SJ, Rodríguez-Enríquez S, Neuzil J, Saavedra E, Moreno-Sánchez R: The causes of cancer revisited: "mitochondrial malignancy" and ROS-induced oncogenic transformation - why mitochondria are targets for cancer therapy. Mol Aspects Med 2010;31:145-170.

64 Connor KM, Subbaram S, Regan KJ, Nelson KK, Mazurkiewicz JE, Bartholomew PJ, Aplin AE, Tai YT, Aguirre-Ghiso J, Flores SC, Melendez JA: Mitochondrial H2O2 regulates the angiogenic phenotype via PTEN oxidation. J Biol Chem 2005;280:16916-16924.

65 Costa R, Peruzzo R, Bachmann M, Montà GD, Vicario M, Santinon G, Mattarei A, Moro E, Quintana-Cabrera R, Scorrano L, Zeviani M, Vallese F, Zoratti M, Paradisi C, Argenton F, Brini M, Calì T, Dupont S, Szabò I, Leanza L: Impaired Mitochondrial ATP Production Downregulates Wnt Signaling via ER Stress Induction. Cell Rep 2019;28:1949-1960.e1946.

66 Morris SL, Huang S: Crosstalk of the Wnt/ $\beta$-catenin pathway with other pathways in cancer cells. Genes Dis 2016;3:41-47.

67 Morris JP, Wang SC, Hebrok M: KRAS, Hedgehog, Wnt and the twisted developmental biology of pancreatic ductal adenocarcinoma. Nat Rev Cancer 2010;10:683-695.

68 Duchartre Y, Kim YM, Kahn M: The Wnt signaling pathway in cancer. Crit Rev Oncol Hematol 2016;99:141149.

69 Zhan T, Rindtorff N, Boutros M: Wnt signaling in cancer. Oncogene 2017;36:1461-1473.

70 Zhang LS, Lum L: Chemical Modulation of WNT Signaling in Cancer. Prog Mol Biol Transl Sci 2018;153:245269. 